\title{
Evolution of the ISM in main-sequence versus starburst galaxies: A motivation for molecular deep fields
}

\author{
Manuel Aravena \\ Núcleo de Astronomía, Facultad de Ingeniería, Universidad Diego Portales \\ Av. Ejército 441, Santiago Chile \\ email: manuel.aravenaa@mail.udp.cl
}

\begin{abstract}
In the last decade, significant progress has been made to understand the evolution with redshift of star formation processes in galaxies. Its is now clear that the majority of galaxies at $z<3$ form a nearly linear correlation between their stellar mass and star formation rates and appear to create most of their stars in timescales of $\sim 1$ Gyr. At the highest luminosities, a significant fraction of galaxies deviate from this main-sequence, showing short duty cycles and thus producing most of their stars in a single burst of star formation within $\sim 100 \mathrm{Myr}$, being likely driven by major merger activity. Despite the large luminosities of starbursts, mainsequence galaxies appear to dominate the star formation density of the Universe at its peak.

While progress has been impressive, a number of questions are still unanswered. In this paper, I briefly review our current observational understanding of this main-sequence vs starburst galaxy paradigm, and address how future observations will help us to have better insights into the fundamental properties of the interstellar medium of these galaxies. Finally, I show recent attempts to conduct molecular deep field observations and the motivation to perform molecular deep field spectroscopy with the Atacama Large Millimeter/submillimeter Array.
\end{abstract}

Keywords. galaxies: evolution, galaxies: high-redshift, galaxies: ISM

\section{Introduction}

One of the most important advances in the understanding of galaxy formation in the last few decades has been the determination of the evolution of the star formation rate (SFR) density of the Universe (e.g. Bouwens et al. 2014 and references therein). The current paradigm implies that galaxies were forming more actively $\sim 8$-10 billion years ago, and they are observed to have quenched their activity by an order of magnitude on average in the local Universe. Thus, the obvious question arises as to why the cosmic star formation density has this behavior, and what is the galaxy population that drives such evolution. Recent galaxy surveys have enabled a much more detailed view of the processes of star formation in large samples.

A major advance has been the determination that the bulk of star forming galaxies at all redshifts define a tight correlation between their stellar mass and their SFRs (Fig. 1; e.g., Brinchmann et al.2004; Elbaz et al.2007; Noeske et al. 2007; Daddi et al. 2007; Pannella et al. 2009; Karim et al. 2011; Rodighiero et al. 2011). Galaxies lying on top of this relation have been termed as "main-sequence" (MS), whereas galaxies lying above this relation on this plane have been called "starbursts". Consequentially, starburst galaxies have higher SFRs than MS galaxies for the same stellar mass (higher specific SFRs). At a fixed stellar mass, starburst galaxies will appear as outliers of the MS, however, as we go to higher masses both populations start to merge (e.g. Rodighiero et al. 2011). 


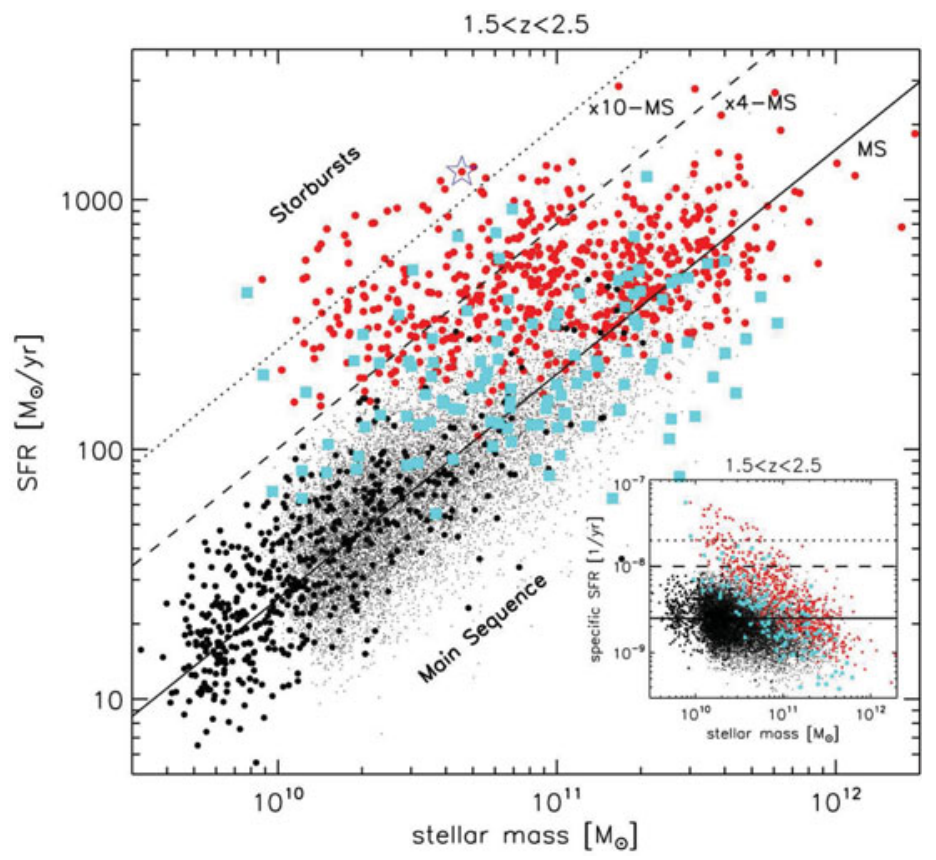

Figure 1. Stellar mass versus SFR for galaxies at $z=1.5-2.5$ (from Rodighiero et al. 2011). Red circles and cyan circles represent IR selected samples from the GOODS South and COSMOS field surveys, respectively. Black filled circles and dots represent $B z K$ color selected galaxies in the same fields, respectively. The solid line represents the MS relation at $z \sim 2$. Color versions of the figures are available online.

The MS has been observed to vary strongly with redshift and also to deviate from linearity at high stellar mass. The redshift evolution takes place mostly on the scaling of the MS, but not in the slope. This means that for the same stellar mass, MS galaxies at higher redshift tend to have higher SFRs. In other words, higher redshift galaxies have higher sSFRs. This increase in sSFRs is in hand with the increase of the cosmic SFR density from $z=0$ to 3 , but also as we will see later on, it goes together with the increase in gas fraction at higher redshifts. Another important result is the determination that the MS is not fully linear with stellar mass, with a flattening at the highest stellar masses. While this behavior is still under active discussion, it is possible that there are mechanisms that inhibit star formation at higher stellar masses (Whitaker et al. 2012; Panella et al. 2015; Lee et al. 2015).

With the advent of large galaxy surveys at infrared wavelengths, it has been possible to quantify the contribution of the different galaxy populations to the SFR density of the Universe. It appears that at $z=2$, the most infrared luminous galaxies $\left(L_{\mathrm{IR}}>10^{12} L_{\odot}\right)$, typically associated with starburst galaxies, have an important contribution. This effect appears to be even more important at higher redshifts. However, more quiescent star forming galaxies tend to dominate at $z<2$ (e.g. Magnelli et al. 2011, 2013; Sargent et al. 2012).

Despite the important progress made in the study of both MS and starburst galaxies with cosmic time, it is still hard to understand the fundamental differences between these populations and driving mechanisms for star formation. For this, we need to understand the star formation process in-situ by studying the raw material from which stars are formed. Namely, the cold molecular interstellar medium (ISM) in these galaxies. 


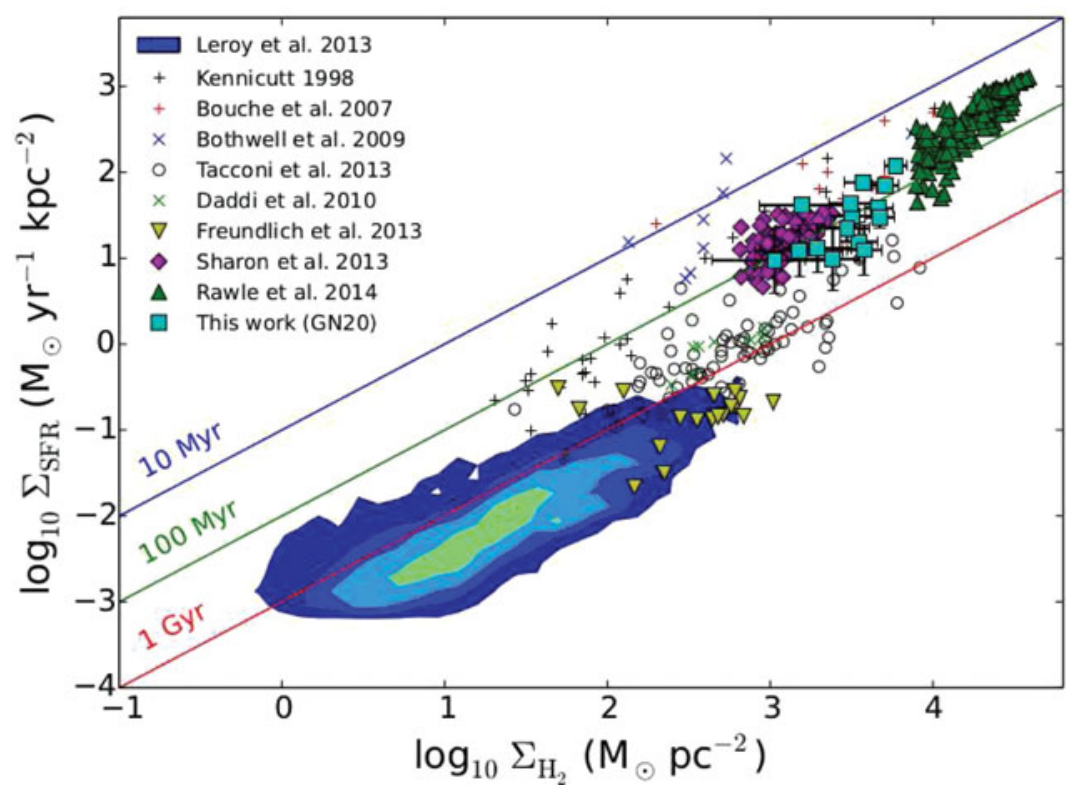

Figure 2. Gas and SFR surface density relationship for various samples of galaxies (from Hodge et al. 2015). Contours indicate the density of local galaxies. Magenta diamonds, cyan squares and green diamonds show the resolved densities for three different submillimeter galaxies (see legend). Notable is the clear separation between samples of MS galaxies and starbursts.

\section{Studying the ISM}

Before continuing, an obvious point should be noted. A typical, massive MS galaxy with a stellar mass of $3 \times 10^{10} M_{\odot}$, has a SFR of about a few tens solar masses per yr. The equivalently massive outlier of the MS will have several hundreds up to a thousand solar masses per yr. In this regime, galaxies will have large IR luminosities $\left(>3 \times 10^{12} L_{\odot}\right)$, and thus will be routinely picked by large-area blank field far-IR and submillimeter surveys of the sky (see review by Casey et al. 2014). Most of the studies of the cold ISM (dust and molecular gas) have so far focused in the study of these "outliers", partly due to the easiness to detect such sources with available submillimeter/radio facilities (see review by Carilli \& Walter 2013).

\subsection{The ISM of starburst galaxies}

A critical measurement to understand how the gas is converted into stars in galaxies is the relationship between their gas masses and SFRs (Fig. 2). Measurements of the dust and cold molecular gas for distant, luminous starburst galaxies (i.e. submillimeter galaxies and quasar host galaxies) indicated that, as observed in local galaxies, this "starformation law" relationship also holds for these objects, spanning for several orders of magnitude (see review Solomon \& Vanden Bout 2005). Such relation implies that these starburst galaxies have gas depletion timescales of $\sim 100 \mathrm{Myr}$, similar to that of local ULIRGs. Another important piece of information to gain further insight into the ISM properties of galaxies is the study of the excitation of the $\mathrm{CO}$ emission, i.e. the relative luminosity ratio of CO lines with different rotational quantum number $(J)$. The specific distribution of this CO spectral line energy distribution (SLED) is sensitive to the density and temperature of the gas. Recently, it has been possible to obtain measurements of the typical CO excitation of SMGs (Bothwell et al. 2013; Spilker et al. 2014). The main conclusion is that starburst galaxies have typical CO SLEDs peaking at $J=5$ or 6 , 
being less extreme than what is found in local ULIRGs or high-redshift quasars. The main difference might come from the different sizes of the emitting regions, as local ULIRGs are shown to be fairly compact.

\subsection{The ISM of main-sequence galaxies}

Recently, numerous measurements of the gas content of MS galaxies have enabled us to determine that these objects follow a different star-formation law than starburst galaxies at similar redshifts (Fig. 2). This difference is visible even if only plotting observable quantities. This implies that MS galaxies have gas depletion timescales of $\sim 0.5-1$ Gyr, which is longer than that observed for starbursts. Another implication of these measurements is that there seems to be a trend for higher-redshift MS galaxies (which have higher SFRs) to have lower gas depletion timescales (e.g. Daddi et al. 2010; Genzel et al. 2010; Sargent et al. 2014). Also, there is a clear increase in the fraction of molecular gas measured from $z=0$ to 2 (Tacconi et al. 2010, 2013; Scoville et al. 2014; Santini et al. 2014; Dessauges-Zavadsky et al. 2015). This rapid increase has been suggested to halt at the higher redshifts $(z>3$; Saintonge et al. 2013), with indications that it may stay constant out to $z=4$ (Bothwell et al. 2013).

Modeling of the ISM in MS galaxies based on line intensity ratios has been very slow and observationally expensive since it requires at least 2 detected CO lines. Currently the number of galaxies with more than one CO line transition detected is very modest (Aravena et al. 2010, 2014; Bolatto et al. 2015; Daddi et al. 2015). The main problem is that as we attempt to observe more typical, less massive galaxies, they become fainter in $\mathrm{CO}$ emission, and thus are hard to detect with current facilities. In particular, obtaining low-J CO measurements for these galaxies is extremely challenging.

Daddi et al. (2015) recently reported the detection of $\operatorname{CO}(J=5-4)$ line emission in 4 main sequence galaxies. All sources showed bright emission, suggesting that the turnover of the CO SLED is happening somewhere at $J=6$ or 7 . On average, these galaxies appear to have gas excitation comparable to that of starbursting submillimeter galaxies, at least out to $J=5$.

\subsection{High resolution studies of molecular gas}

High resolution molecular gas studies hold the key to understand the fundamental differences between starbursts and main sequence galaxies at all redshifts. Models predict that starbursts galaxies should show features typical of galaxy mergers, with the molecular gas and dust concentrated within a few kpc and dispersion dominated kinematics. Main sequence galaxies should show an extended disk with rotation dominated kinematics and several gas clumps within this disk. In spite of that, the number of resolved studies are still modest, being mostly focused in single source studies. These show important differences from source to source, with disturbed dynamics in some cases (Sharon et al. 2013) and rotation dominated dynamics in others (Hodge et al. 2015).Current work shows that it is possible to dissect the high redshift galaxies and resolve the star formation law relations within these galaxies (Fig. 2). These studies indicate that starbursting submillimeter galaxies tend to follow a different scaling in the star formation law, with higher SFR surface densities compared to main sequence galaxies (e.g. Freundlich et al. 2013; Sharon et al. 2013; Rawle et al. 2013; Hodge et al. 2015).

Despite the substantial progress, resolved observations are limited by the faintness of the CO line emission. Resolving the star forming activity and molecular gas (CO) in distant starbursting galaxies requires significant amounts of observing time, even in the era of the Atacama Large Millimeter/submillimeter Array (ALMA). This is accentuated when attempting to observe more "normal" galaxies, which are fainter. Therefore, 

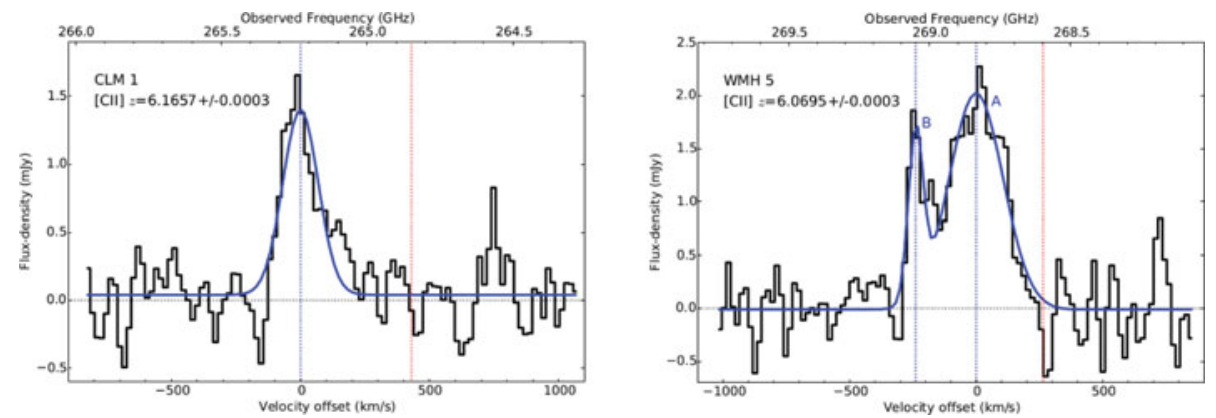

Figure 3. [CII] emission line spectra toward two typical galaxies at $z=6$ (Willott et al. 2015). The recent detections of [CII] emission in several $z \sim 5-6$ galaxies has opened a remarkable opportunity to the study of star formation at the end of the cosmic reionization era.

acquisition of resolved dust and $\mathrm{CO}$ observations in statistical samples of distant star forming galaxies could be seriously limited. A solution used to overcome this limitation has been to take advantage of the amplification provided by gravitational lensing. However, such approach makes it complicated to interpret the emission given the necessity to model the foreground lens.

\subsection{How to access the $z>4$ Universe?}

As mentioned above, detecting CO line emission in normal galaxies is difficult even at $z=2-3$ (e.g. Magdis et al. 2012). At $z>4$, the current and most successful approach has been to target a brighter line, specifically the [CII] emission line. Observationally, this line has a rest-frame frequency of $\sim 1900.5 \mathrm{GHz}$, and thus is redshifted into the 380-210 $\mathrm{GHz}$ regime at $z=4.0-8.0$. Most importantly, this line is a dominant coolant of the ISM in galaxies, typically making $0.1-1 \%$ of the integrated IR luminosity. Furthermore, this line has been found to trace both photon dominated and HII regions in galaxies, being directly linked to the process of star formation (e.g. Carilli \& Walter 2013).

Since the [CII] constitutes a critical tool for the study of the most distant galaxies, it has been widely used for this purpose. After several attempts to detect the [CII] line emission at $z>4$ from normal galaxies (e.g. Lyman- $\alpha$ emitters and Lyman Break Galaxies), the unparalleled sensitivity of ALMA allowed us to detect this line in a number of galaxies at $z \sim 5-7$ (Fig. 3; e.g. Riechers et al. 2014; Capak et al. 2015; Willott et al. 2015; Maiolino et al. 2015).

Typically, galaxies at $z>4$ are selected using the optical dropout technique, based on the identification of the Lyman break in the UV spectra. To confirm and obtain spectroscopic redshifts it is thus necessary that the Lyman- $\alpha 1216 \AA$ emission line is sufficiently strong. However, it has been seen that the Lyman- $\alpha$ emission can be strongly suppressed by the intergalactic medium at $z>6$. Furthermore, for objects at $z>6.5$, this line is redshifted into a part of the spectrum that is contaminated by atmospheric emission, making it even more difficult to detect the Lyman- $\alpha$ from the ground. The recent detection of [CII] emission in distant galaxies suggest it may be possible to directly obtain spectroscopic redshifts based on this line, and also blindly identify such galaxies in deep field spectroscopic observations with ALMA.

\section{Unbiased views of the cosmic evolution of the ISM}

The star formation activity is necessarily linked to the molecular gas content at all redshifts (star formation law). The steep increase in the SFR density of the Universe 


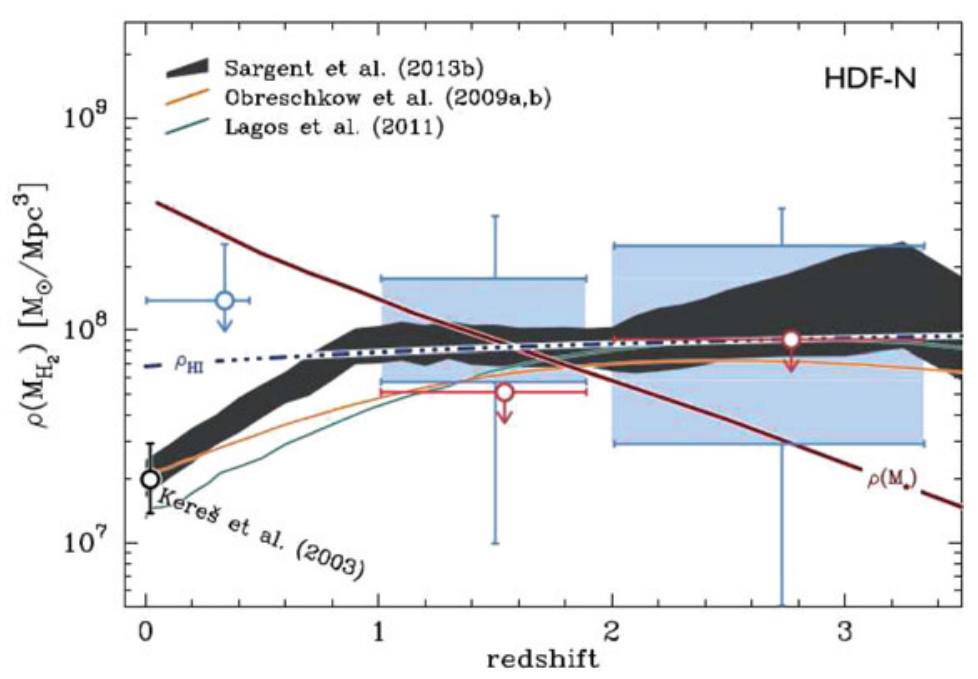

Figure 4. Evolution of the cosmic molecular gas mass density (from Walter et al. (2014)). The orange and green curves show the predictions from semi-analytical cosmological models. The grey shaded area show semi-empirical predictions from Sargent et al. (2014). The blue shaded area show the current limits based on PdBI observations of the Hubble Deep Field North. Deeper ALMA observations are needed to provide better constraints to this evolution.

between $z=0$ to 2 appears to relate to the increase in molecular gas fractions in this redshift regime (i.e. the fraction of material that is available to sustain star formation). It thus follows that the evolution of the SFR density may be linked to a more fundamental quantity, the cosmic density of the molecular gas (the amount of molecular gas per comoving volume) across times.

It must be noted that most of what we know so far about the ISM properties of distant galaxies has been biased due to pre selection of sources in optical images which trace the stellar and star forming components of galaxies. What we need are deep wideband molecular spectroscopic surveys over significant cosmic volumes, in order to directly pick up the cold gas phase of galaxies and select galaxies in this way.

A pilot study has been recently performed with the Plateau de Bureau Interferometer (PdBI) by Decarli et al. (2014) and Walter et al.(2014). See Figure 4. They conducted deep single pointing covering the full $3 \mathrm{~mm}$ window, and thus covering the most relevant redshift ranges for $\mathrm{CO}$ line emission $(z<0.5, z=2-6)$. Based on the number of detected sources at different CO luminosities, they were able to put constraints on the CO luminosity functions at three different redshifts, and thereby constrain the cosmic density of molecular gas with redshift. While this study could only provide rough constraints, their results provide the first insight into the relationship between the cosmic densities of star formation and molecular gas. To provide further constraints we obviously need to go deeper and cover larger areas. The only way to achieve this is using ALMA. Deep millimeter spectroscopic observations of large cosmological volumes with ALMA will be critical to provide an unbiased census of the cold gas phase of galaxies at all redshifts. As in Decarli et al. (2014), ALMA spectroscopy covering the full 3-mm window will enable us to target the CO line emission at $z>2$. ALMA band 6 measurements, covering the full $1-\mathrm{mm}$ window, will allow us to cover precisely the redshift range $(z<2)$ that is not constrained by the band 3 observations for CO line emission, making simultaneous observations of both bands and the same region of the sky highly complementary. Most 
importantly, ALMA band 6 observations will cover the [CII] line emission for galaxies at $z=6-8$.

\section{Concluding remarks}

Despite the large IR luminosities of starburst galaxies, MS galaxies appear to dominate the evolution of the SFR density of the Universe out to $z=2$. MS galaxies seem to be subject to a different star forming law, suggesting that the mechanisms in which star formation is taking place in these galaxies differs from that of starbursts. The best way to characterise such mechanisms is by studying the gas and dust that form the ISM of galaxies.

Despite the great advances made by studies of the ISM properties of both starburst and MS galaxies at high redshift, the number of detections of molecular gas is still scarce, and there is a particular need for resolved observations of gas and dust. The advent of ALMA brings forth the hope that such observations could become accessible in larger samples in the near future, even though it will still require significant investments of observing time. Finally, deep field molecular line spectroscopy with ALMA over considerable volumes promises to provide a critical census of the molecular gas content and properties in galaxies out to $\mathrm{z}=8$.

\section{References}

Aravena, M. et al. 2010, ApJ, 718, 177

Aravena, M. et al. 2014, MNRAS, 442, 558

Bolatto, A. et al. 2015, ApJ, 809, 175

Bothwell, M. et al. 2013, MNRAS, 429, 3047

Bouwens, R. et al. 2014, ApJ, 795, 126

Brinchmann, J. et al. 2004, MNRAS, 351, 1151

Capak, P. et al. 2015, Nature, 522, 455

Carilli, C. L. \& Walter, F. 2013, ARA\&A, 51, 105

Daddi, E. et al. 2007, ApJ, 670, 156

Daddi, E. et al. 2010, ApJ (Letters), 714, 118

Daddi, E. et al. 2015, A\& $A, 577,46$

Decarli, R. et al. 2014, ApJ, 782, 78

Dessauges-Zavadsky, M. et al. 2015, A\& A, 577, 50

Elbaz, D. et al. 2007, A\&A, 468, 33

Freundich, J. et al. 2013, A\& $A, 553,130$

Genzel, R. et al. 2010, MNRAS, 407, 209

Karim, A. et al. 2011, ApJ, 730, 61

Magnelli, B. et al. 2011, A\&A A, 528, 35

Magnelli, B. et al. 2013, A\&SA, 553, 132

Maiolino, R. et al. 2015, MNRAS, 452, 54

Noeske, K. G. et al. 2007, ApJ, 660, 47

Pannella, M. et al. 2009, ApJ, 698, 116

Riechers, D. et al. 2014, ApJ, 796, 84

Rodighiero, G. et al. 2011, ApJ, 739, 40

Saintonge, A. et al. 2013, ApJ, 778, 2

Santini, P. et al. 2014, A\&A, 562, 30

Sargent, M. T. et al. 2014, ApJ, 793, 19

Sargent, M. et al. 2012, ApJ, 747, 31

Scoville, N. et al. 2014, ApJ, 783, 84

Solomon, P. \& Vanden Bout 2005, ARA\&A, 43, 677 
Spilker, J. S. et al. 2014, ApJ, 785, 149

Tacconi, L. et al. 2013, ApJ, 768, 74

Tacconi, L. et al. 2010, Nature, 463, 781

Walter, F. et al. 2014, ApJ, 782, 79

Willott, C., Carilli, C. L. \& Wagg, J. 2015, ApJ, 807, 180 